\title{
LANGUAGE DISORDER AND POLYMICROGYRIA
}

The co-occurrence of developmental language disorder and reading impairment in members of three families with perisylvian polymicrogyria is reported from the State University of Campinas, and University of Sao Paulo, Brazil. The severity of language impairment correlated with the extent of the polymicrogyria, patients with the worst language deficit having diffuse bilateral perisylvian polymicrogyria while patients with mild impairment showing subtle MRI anomalies. (Oliveira EPM, Hage SRV, Guimaraes CA et al. Characterization of language and reading skills in familial polymicrogyria. Brain Dev April 2008;30:254-260). (Respond: Dr MM Guerreiro: E-mail: mmg( $\left(\begin{array}{l}\text { fcm.unicamp.br). }\end{array}\right.$

COMMENT. Polymicrogyria is a cerebral developmental anomaly, characteristically perisylvian in location, giving the cortical surface a pebbled, 'chestnut kernel,' or 'Moroccan leather' appearance. Genetic or acquired causes are described. A causative diagnosis was established in 20 of 48 cases recently reported (de Wit MCY et al. Arch Neurol March 2008;65:358-366; Ped Neur Briefs March 2008;22:24). A genetic cause was suspected in 6 patients with multiple congenital abnormalities and in 4 with consanguineous parents or multiple affected family members. A gestational insult was the probable cause in 7 patients. Polymicrogyria can be localized or diffuse, unilateral or bilateral. The cortex is thickened, without recognizable layers or with 4 layers in place of the usual 6 . The brain stem may be hypoplastic, especially involving the pyramidal tracts. In severe cases, the child has spastic diplegia or hemiplegia, mental retardation, and seizures. The MRI has permitted the diagnosis and recognition of milder forms of polymicrogyria, some associated with language and reading disorders, as described in the above study.

\section{NONAUTISTIC MOTOR STEREOTYPIES}

Clinical features and long-term outcomes of 100 children ( 62 boys and 35 girls) with motor stereotypies were evaluated by review of records and telephone interviews at Johns Hopkins Hospital, Baltimore, MD. Mean age was $8.3+/-4.5$ years. Age at onset was $<24$ months in $81 \%$. All children were in a regular classroom and were at least grade $\mathrm{C}$ in achievement. Six had a history of early language delay. Repetitive, rhythmic, involuntary movements consisted of finger wiggling and/or flapping of hands or arms; $20 \%$ also exhibited facial grimacing, and $8 \%$ had head nodding movements. Movements occurred once a day or more in $90 \%$ and lasted less than a minute in $62 \%$. Triggers included excitement/happiness in $80 \%$, anxiety and stress in $26 \%$, and fatigue in $21 \%$. Stereotypies ceased during sleep and when cued by calling his or her name. Family history was positive for most motor stereotypies in 17\% first-degree relatives, but negative in patients with head nodding. Associated conditions included ADHD in $30 \%$, tics in $18 \%$, and OCD in $10 \%$. Various medications prescribed in 20 patients, including clonidine, risperidone, and oxcarbazepine, were ineffective, and behavior modification in 14 resulted in modest improvements in 5 patients. Follow-up ranged from 2 months to 26 years, with a median of 6 years. Movements were persistent in 94 children, continuing for $>10$ years in $22 \%$, and $6-10$ years in $44 \%$. Prognosis was better in children with head nodding than in those with hand/arm movements; head nodding resolved in one third, compared to only $3 \%$ with hand movements $(\mathrm{P}=0.001)$. (Harris KM, Mahone EM, Singer HS. Nonautistic motor stereotypies: 
clinical features and longitudinal follow-up. Pediatr Neurol April 2008;38:267-272). (Dr Singer, Division of Pediatric Neurology, Department of Pediatrics, Johns Hopkins School of Medicine, Child Health Building, $200 \mathrm{~N}$ Wolfe St, Suite 2158, Baltimore, MD 21287).

COMMENT. The present findings in 100 patients are similar to those reported in a previous study of 40 patients from the same institution (Mahone EM et al. J Pediatr 2004;145:391-395; Ped Neur Briefs Sept 2004;18:72). Most motor stereotypies are chronic and persistent and of greater concern to parents and physicians than to the child. Approximately $50 \%$ of patients with motor stereotypies $>7$ years of age have a comorbid disorder such as ADHD, tics or OCD.

Motor stereotypies are defined as involuntary, bilateral, repetitive, rhythmic movements associated with periods of excitement, stress, and fatigue. (Castellanos FX et al. J Clin Psychiatry 1996;57:116-122). They are common in mentally retarded and autistic children, and less prevalent in otherwise normal, healthy children. Associated disorders such as tics are distinguished by a later age of onset, 5-10 years, their asymmetry, vocal as well as motor, and response to medication.

\section{NEUROMUSCULAR DISORDERS}

\section{CONGENITAL FIBER TYPE DISPROPORTION GENETICS}

Novel heterogeneous missense mutations in five families with congenital fiber type disproportion (CFTD) were identified in a study at Children's Hospital at Westmead, University of Sydney, and other centers in Australia, Canada, and France. In 11 affected patients with TPM 3 gene mutations and CFTD, symptoms of hypotonia presented in the first year. Some had a "dropped head" posture while crawling. Five walked late at $18-60$ months, while 6 walked at a normal age. Most improved functionally until adolescence, when motor ability stabilized or slowly declined. Four patients older than 30 years were still ambulant. Respiratory insufficiency occurred during sleep, despite good limb strength, and ventilatory support was required as early as 3.5 years in one patient and as late as 55 years in one. One died unexpectedly at 45 years old. Spinal changes were invariable, with lumbar lordosis and thoraric kyphosis in early childhood, becoming more severe in late childhood or adulthood. Neck muscle weakness and extensor contractures were common. Most had generalized amyotrophy, proximal limb weakness, and a waddling gait. Mild facial weakness and ptosis, and winged scapula were common. Intellectual function was normal. Cardiac function was normal, except for 1 patient with left ventricular hypertrophy. CK level was low normal and rarely, mildly increased. Nerve conduction studies were normal, and EMG normal or myopathic. In muscle biopsies, type 1 fibers were atrophied and $50 \%$ smaller than type 2 fibers. Type 2 fibers were hypertrophied, 1.6 times normal diameter, and $25 \%$ had internal nuclei. In a sixth family with TPM3 mutation, some patients had features of CFTD and others had nemaline myopathy. TPM3 mutation is the most common cause of CFTD in reported cases. (Clarke NF, Kolski H, Dye DE et al. Mutations in TPM3 are common causes of congenital fiber type disproportion. Ann Neurol March 2008;63:329-337). (Respond: Prof Kathryn N North, Children's Hospital at Westmead, Locked Bag 4001, Westmead, NSW 2145, Australia. E-mail: kathryn(a,chw.edu.au). 